\title{
Lumped Arrays of Shunted Bicrystal Josephson Junctions
}

\author{
Alexander M. Klushin, Konstantin S. Il'in, Michael Siegel, Marco Schubert, Gerd Wende, and Hans-Georg Mayer
}

\begin{abstract}
We report on the design, fabrication, and investigation of lumped arrays of Josephson junctions embedded into coplanar strip-lines (CPS). The arrays were incorporated into the CPS by meandering a bilayer across the grain boundary. Limitations on junctions packing density are discussed. The dependence of the length of the lumped array on spread of critical currents of junctions in the array was found. The investigated arrays with 431 junctions have shown frequency locking with an external irradiation. Voltage steps up to $20 \mathrm{mV}$ at liquid nitrogen temperature were demonstrated.
\end{abstract}

Index Terms-Arbitrary waveform, arrays, Josephson junctions, superconducting devices, voltage standard.

\section{INTRODUCTION}

C ONSIDERABLE recent attention has been focused on the development of a quantum-mechanically accurate voltage source for both ac and dc voltage metrology [1]. This voltage source is based on pulse-driven arrays of nonhysteretic Josephson junctions. For appropriate operation every pulse specified by a digital code has to bias every junction in the array without failures. Such an operation mode can be realized for example in lumped arrays. Recently, in a circuit with more than $7000 \mathrm{Nb}-\mathrm{PdAu}-\mathrm{Nb}$ junctions an output ac voltage greater than $100 \mathrm{mV}$ was achieved in the frequency range from $1 \mathrm{kHz}$ to $50 \mathrm{kHz}$ [2].

Non-hysteretic junctions are naturally available in high-temperature superconductor (HTS) technology. Series arrays of shunted $\mathrm{YBa}_{2} \mathrm{Cu}_{3} \mathrm{O}_{7}$ (YBCO) bicrystal Josephson junctions were used to generate dc voltages at elevated temperatures [3]. Recently, quantum voltages of an array of YBCO bicrystal junctions were calibrated against a programmable Josephson array voltage standard of the Physikalisch-Technische Bundesanstalt [4]. The coincidence of quantum voltages of the array of HTS junctions at $64 \mathrm{~K}$ and of the array of niobium junctions at $4.2 \mathrm{~K}$ was measured with an uncertainty of 1.7 parts in $10^{8}$. This result has shown that arrays of bicrystal junctions operate properly and can be used for high-precision measurements. At the same time, these junctions have large critical currents, $I_{c}$ and first current steps, $\Delta I_{1} \approx 1 \mathrm{~mA}$, at temperatures up to $80 \mathrm{~K}$, and provide stability against noise as well as a large

Manuscript received August 5, 2002. This work was supported by the German BMBF under Grant 13N7534/1.

A. M. Klushin, K. S. Il'in, and M. Siegel are with Institut für Schichten und Grenzflächen, Forschungszentrum Jülich GmbH 52425, Germany (e-mail: a.klushin@fz-juelich.de).

M. Schubert, G. Wende, and H.-G. Mayer are with the Department of Cryoelectronics, Institute for Physical High Technology, Jena D-07702, Germany (e-mail: marco.schubert@adam.ipht-jena.de).

Digital Object Identifier 10.1109/TASC.2003.813960 output current [5]. It means that arrays with small junctions and high package density can be produced. Also characteristic voltages, $V_{c}=I_{c} R$ ( $R$ is the junction resistance), can be varied in a practically important range between $20 \mu \mathrm{V}$ and $200 \mu \mathrm{V}$ enabling operation in a broad band of frequencies. An elevated operation temperature of HTS arrays will allow the use of short transmission lines with low distortions of the output ac voltage. A small and low-cost cryogenic system could be very important for practical applications.

We focus on design, fabrication, and investigation of lumped arrays of Josephson junctions embedded into coplanar striplines.

\section{HTS ARRays With High Package Density}

A large number of junctions per chip is required to generate output voltages of sufficient magnitude for use in metrological applications. We realize our series arrays of bicrystal junctions using a meander line crossing the grain boundary (GB) of the substrate many times. There are two possibilities for enhancing the packing density of the junctions. One is to use substrates with more than one GB. The grain boundaries are parallel and spaced only about $10 \mu \mathrm{m}$ to $20 \mu \mathrm{m}$. Thus, the meander can cross all of them, resulting in a $n$-times enhanced junction density per unit length, if $n$ is the number of grain boundaries. Arrays prepared on such substrates with $n=2$ showed promising results and were described in [6].

Another option is to push the meanders together [7]. While the width of the junctions is determined by optimal junction size considerations and cannot be reduced, the space between junctions needs to be as small as possible. Therefore, a patterning process is needed which allows patterning of narrow trenches even into thick YBCO-Au bilayers.

\section{A. Electron-Beam Lithography}

We applied a three-level mask technique together with electron-beam lithography, similar to the technique described in [7], [8].

Shunted bicrystal junctions were fabricated using Au-YBCO bilayers [9] deposited in situ on symmetrical yttrium-stabilized zirconium oxide (YSZ) substrates with misorientation angle of $\Theta=24^{\circ}$. The thickness of YBCO and Au films were $0.3 \mu \mathrm{m}$ and $0.6 \mu \mathrm{m}$, respectively.

The meander-like sub- $\mu \mathrm{m}$ structure was fabricated by the electron beam lithography and etching technique as shown in Fig. 1. First we covered the YBCO-Au double layer with a 500 $\mathrm{nm}$ thick $\mathrm{Nb}$ film using dc magnetron sputtering. On top of the $\mathrm{Nb}$ layer a $d=20 \mathrm{~nm}$ thick gold layer was deposited in situ by 


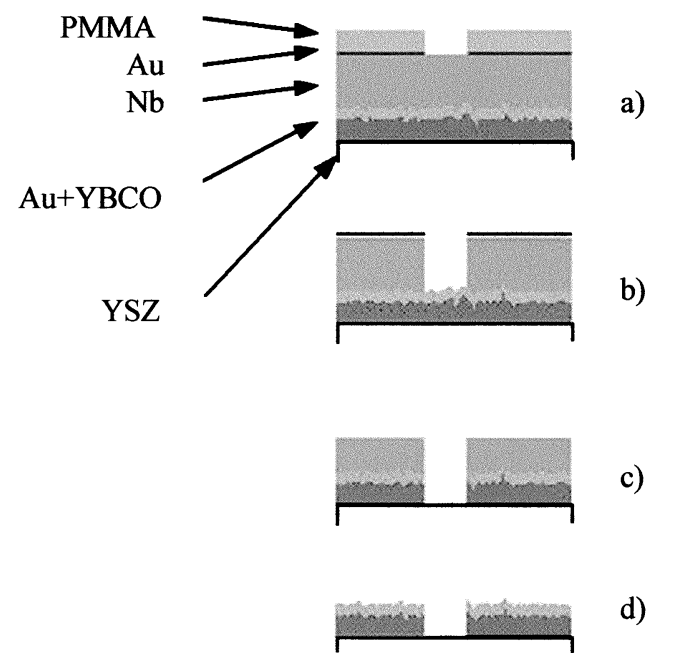

Fig. 1. Three level mask for sub- $\mu \mathrm{m}$ lithography and sequence of the etch steps.

rf magnetron sputtering. The meander structure was created in the electron-beam resist PMMA 950 with a thickness of about $150 \mathrm{~nm}$ [Fig. 1(a)]. We used E-beam lithography in a $1000 \times$ $1000 \mu \mathrm{m}^{2}$ writing field at $5 \mathrm{KV}$ electron energy and $20 \mathrm{pA}$ electron-beam current. The total length of the meander-like structure was up to $8 \mathrm{~mm}$, depending on the required number of junctions. The gold structure obtained in this way was used as a mask for patterning the $\mathrm{Nb}$ layer by reactive ion etching (RIE) [Fig. 1(b)]. We transferred the resulting layout into the double layer by Ion-Beam Etching (IBE) using the Nb layer as a mask [Fig. 1(c)]. Finally, residual $\mathrm{Nb}$ was removed from the top of the YBCO-Au structure by RIE [Fig. 1(d)]. Using this technique, arrays of bicrystal junctions with a width $w$ from 0.5 $\mu \mathrm{m}$ to $4 \mu \mathrm{m}$ and a distance between junctions in the meander array from $0.3 \mu \mathrm{m}$ to $1 \mu \mathrm{m}$ were obtained.

\section{B. Effect of Width on Spread of Junctions Parameters}

Eight 100-junctions arrays with $w$ equal to $2 \mu \mathrm{m}, 1 \mu \mathrm{m}, 0.7$ $\mu \mathrm{m}$ and $0.5 \mu \mathrm{m}$ have been explored. Specially designed leads allowed us to measure each 10 junction sub-array of a 100 junctions array. It was found, that the critical temperature of superconductive strips without GB was $87 \mathrm{~K}$ to $89 \mathrm{~K}$ and was practically independent of $w$. The critical current density of these strips was typically larger than $1 \times 10^{6} \mathrm{~A} / \mathrm{cm}^{2}$ at $77 \mathrm{~K}$ and also independent of the strip width.

To evaluate the influence of the width on spread of the normal resistance $\delta R=\left(R_{\max }-R_{\min }\right) / R_{\min }$ we used the method suggested earlier [10]. Here, $R_{\max }$ and $R_{\min }$ are the maximal and minimal resistance of junctions connected in series, respectively.

To make a conclusion about the value of $\delta R$ we measured the mean normal resistance $R_{s a}$ in each 10-junctions sub-arrays. After determining $R_{s a, \max }$ and $R_{s a, \text { min }}$ we have calculated the spread of the mean values of $R_{s a}$ among sub-arrays, $\delta R_{s a}$. Then we estimated the spread among individual junctions as $\delta R \approx$ $\delta R_{s a} \times \sqrt{M}$, where $M=10$ is the number of junctions in each sub-array. As shown in Fig. 2 the mean value of the resistance in the array increases inversely with the junction width. The spread

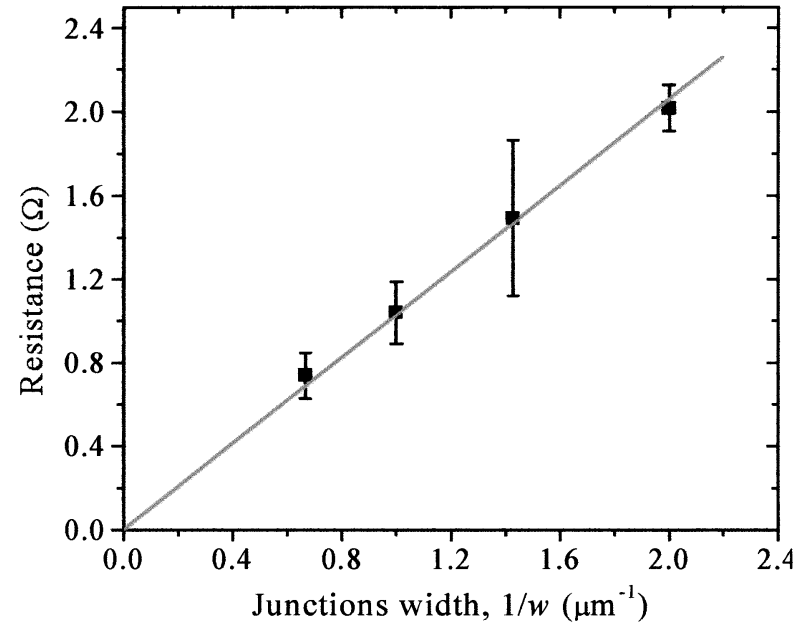

Fig. 2. Dependence of resistance of junctions on inverse junctions width. Bars represent $1-\sigma$ standard deviations and line is the linear least square quadrate fit.

of the junction resistance with $w \approx 2 \mu \mathrm{m}$ is close to the typical value of $15-20 \%$ measured on arrays of junctions with $w \geq$ $3 \mu \mathrm{m} \mathrm{[10].} \mathrm{Unfortunately,} \mathrm{the} \mathrm{spread} \mathrm{of} \mathrm{resistance} \mathrm{increased}$ rapidly with $1 / w$. At the same time the spread of the critical currents $\delta I_{c}$ drastically increases in comparison with junction's width $w \geq 3 \mu \mathrm{m}$. Both these effects hinder an application of bicrystal junctions with $w<2 \mu \mathrm{m}$ in large series arrays. The mechanism of such behavior of bicrystal junctions parameters was discussed in detail previously [11].

\section{Effect of Width on Junctions Parameters}

For large operating margins of the quantum voltage source the amplitude of the first voltage step should be of the same order as the critical current, $\Delta I_{1} \approx I_{c}$. This is possible if $I_{c} R \approx \Phi_{0} f$, where $\Phi_{0}$ is the magnetic flux quantum and $f$ is a frequency of the sine-wave-bias current. In pulse-driven arrays optimum operation conditions occur when $f$ is equal to $3 / 2 f_{s}$ or $5 / 2 f_{s}$, where $f_{s}$ is the clock frequency [12]. The typical value of $f_{s} \approx 5$ $\mathrm{GHz}$ determines $f \approx 10 \mathrm{GHz}$ and $V_{c} \approx 20 \mu \mathrm{V}$. At the same time, a step amplitude larger than $1 \mathrm{~mA}$ is desirable to ensure stability against thermal noise at liquid nitrogen temperatures. To meet these conditions, the shunted resistance of junctions should be smaller than $R \approx 0.02 \Omega$.

For estimation of obtainable junctions parameters, we took into account that the critical current density at $77 \mathrm{~K}$ is equal to 5 $\times 10^{4} \mathrm{~A} / \mathrm{cm}^{2}$ for YBCO GB junctions fabricated on substrates with symmetrical $\mathrm{GB}$ and $\theta=24^{\circ}$ [5]. It means that for a film thickness of $d \leq 1 \mu \mathrm{m}$ and width of $w=1 \mu \mathrm{m}$ the critical current $I_{c}$ will be not larger than $0.5 \mathrm{~mA}$ at a temperature of $T=77 \mathrm{~K}$.

Another important parameter, the shunt resistance of junctions, can be found from [13]

$$
R_{s}=\frac{1}{w} \sqrt{\frac{\rho_{v} \rho_{c}}{d_{n}}} .
$$

Here, $\rho_{v}$ and $\rho_{c}$ are the specific resistance of the gold film with a thickness $d_{n}$ and contact resistance between gold and YBCO films, respectively. For typical values of $\rho_{v}=5 \times 10^{-7} \Omega \mathrm{cm}$ and $\rho_{c}=10^{-8} \Omega \mathrm{cm}^{2}$ and a thickness of the Au film $d_{n}=$ 


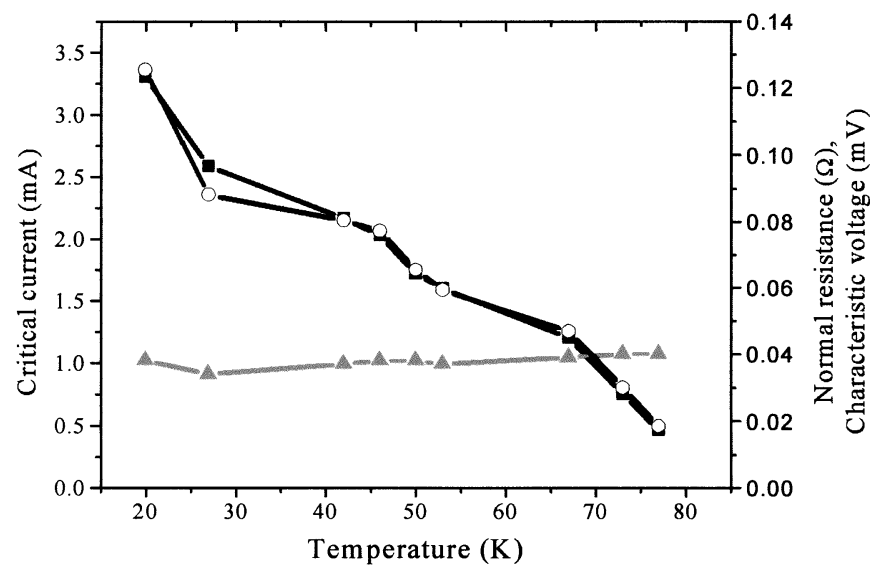

Fig. 3. Dependence of the critical current ( $\square$, left scale), characteristic voltage and resistance of shunted junctions $(\bigcirc, \boldsymbol{\Lambda}$, right scale) on temperature. Lines are drawn to guide the eye.

$0.5 \mu \mathrm{m}$ the shunt resistance will be equal to $R=0.1 \Omega$ for $w=$ $1 \mu \mathrm{m}$. This gives a large characteristic voltage of $V_{c} \leq 50 \mu \mathrm{V}$.

As shown in Fig. 3 the critical current can be increased by decreasing the operating temperature. Unfortunately, the resistance of the shunted junctions remains constant in spite of the presence of a normal metal film on top of the HTS film. Therefore decreasing temperature is accompanied by a further increase of $V_{c}$. To meet the conditions essential for proper operation of the pulse-driven arrays at a sine frequency of $f \approx 10$ $\mathrm{GHz}$ the junction width should be increased.

On the another hand, when the physical width of the junction becomes comparable with $4 \lambda_{j}\left(\lambda_{j}\right.$-Josephson penetration depth), the total critical current is no longer linearly proportional to the junction area and a much larger microwave power must be applied in order to reach the maximal step height. In our case, $\lambda_{j}$ is approximately equal to $1 \mu \mathrm{m}$ for a critical current $I_{c} \approx 1$ $\mathrm{mA}$.

A reasonable width of junctions from $4 \mu \mathrm{m}$ to $6 \mu \mathrm{m}$ with YBCO and Au films thickness from $0.5 \mu \mathrm{m}$ to $1 \mu \mathrm{m}$ will allow the junctions the conditions essential for proper operation of the pulse-driven arrays at sine frequency $f \approx 10 \mathrm{GHz}$ are be met. An increase of $f$ will lead to a further increase of the packing density of bicrystal junctions.

\section{MicrowaVe PROPERTIES OF LUMPED ARRAYS}

For uniform distribution of the microwave bias current the length $L$ of the electrically small array should be a factor $m$ shorter than the effective wavelength $\lambda_{\text {eff }}$. The magnitude of $m$ depends on distribution of current in the microwave line and is minimized by placing the array close to the current loop. It is reasonable that the spread of the microwave current is of order $\delta I_{c}$. It can be shown that in this case [14]

$$
L<\frac{\lambda_{e f f}}{\pi} \sqrt{\frac{1-\delta I_{c}}{2}} .
$$

For example, for a typical value of $\delta I_{c} \approx 0.5[10]$ and dielectric constant of YSZ $\varepsilon=26$ the array with a length of $L=1.3$ $\mathrm{mm}$ can be considered as lumped up to about $10 \mathrm{GHz}$. Over this length 200-250 bicrystal junctions can be placed along one GB.

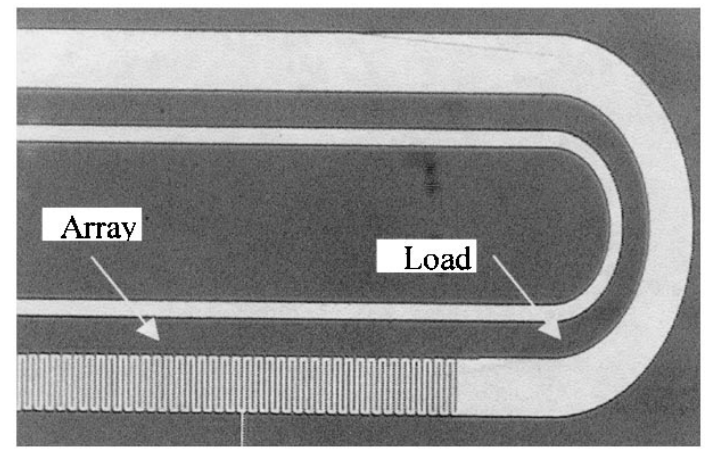

Fig. 4. Photograph of the part of a CPS with an embedded array of bicrystal junctions.

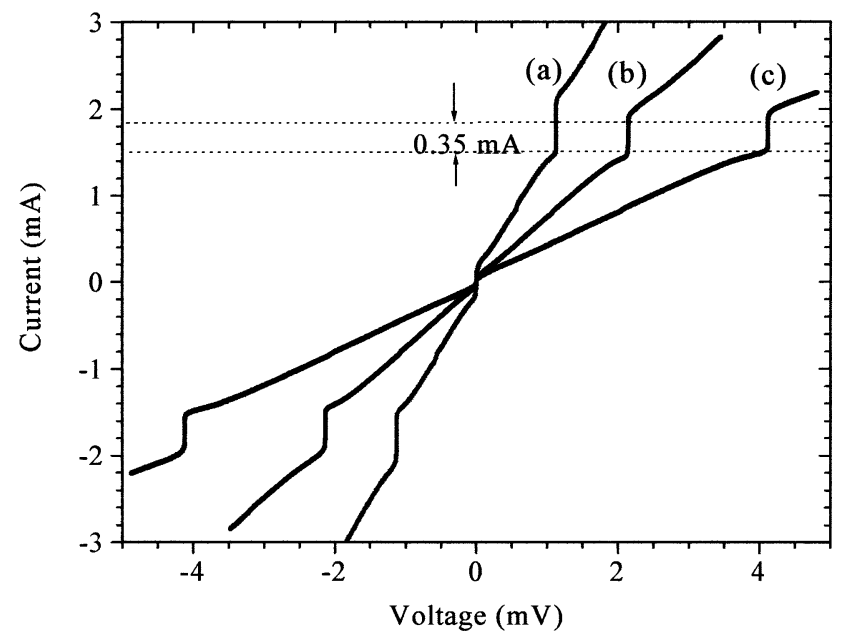

Fig. 5. Current-voltage characteristics of a lumped array under microwave irradiation at a frequency $f=31101 \mathrm{MHz}$ : (a) 16 junctions, (b) 32 junctions and (c) 64 junctions at $T=78 \mathrm{~K}$

For microwave irradiation the array of HTS junctions was embedded in the coplanar transmission line [15] as it is shown in Fig. 4. The CPS with a characteristic impedance of about 50 $\Omega$ consisted of $4 \mu \mathrm{m}$ and $12 \mu \mathrm{m}$ wide strips separated by a slot of $8 \mu \mathrm{m}$ wide. As a load a long part of the same CPS was used.

Long arrays containing more than 1700 junctions were embedded in a $12 \mu \mathrm{m}$ wide strip, as shown in Fig. 4. The junctions' width was $3 \mu \mathrm{m}$ and the distance between the junctions was 1 $\mu \mathrm{m}$. Thin film current leads allowed independent dc-bias and voltage measurements in separate parts of the array. These leads disturb an uniform microwave distribution along the full array. Consequently a large standing wave was observed.

In this case only a portion of an array with a length of about $L$ can be synchronized. Indeed, we have measured voltage steps on sub-arrays containing 16, 32 and 64 junctions respectively (Fig. 5). The critical current and normal resistance of these segments were $I_{c}=0.7 \mathrm{~mA}$ and $R=0.04 \Omega$, respectively. All 112 junctions were spaced over a distance of $0.5 \mathrm{~mm}$. From the (2) this sub-array can be considered as lumped up to $35 \mathrm{GHz}$.

In another array with the same parameters, but having only three dc pads, the uniformity of the microwave-current distribution was much better. In this case the sub-array which contains 431 junctions was synchronized under microwave irradiation with a frequency of $f=22731 \mathrm{MHz}$. A Shapiro step at a 


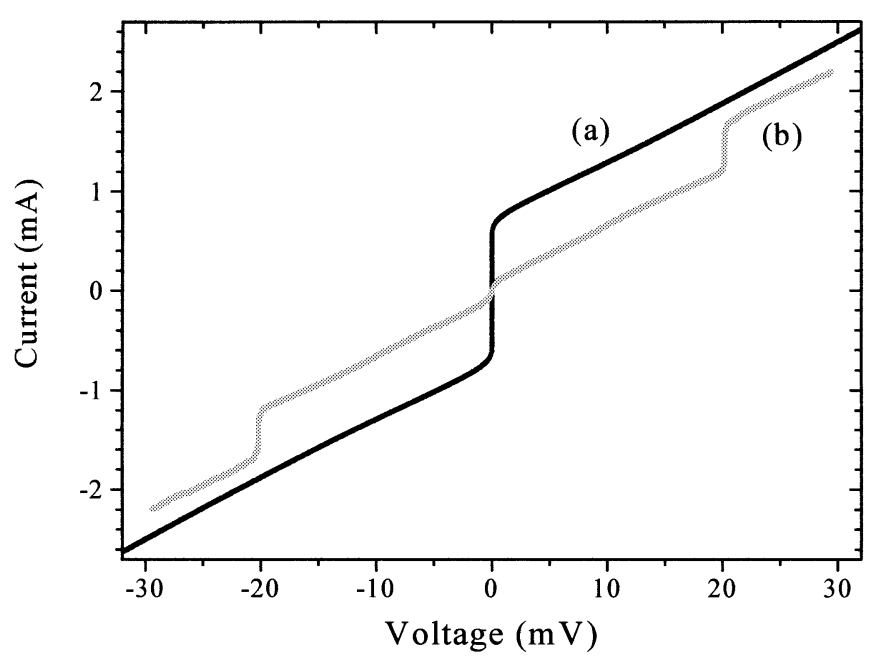

Fig. 6. Current-voltage characteristics for a series array of 431 junctions at $T=78 \mathrm{~K}$. (a) without microwave power and (b) with microwave power.

voltage of about $20 \mathrm{mV}$ was observed, as shown in Fig. 4. This result represents a synchronization in the distributed array, because its length was much larger than the value of $L$ calculated from (2).

\section{CONCLUSION}

Pulse-driven, electrically short arrays are promising for application in the new generation of ac and dc quantum voltage sources. An electron-beam lithography process for fabrication of arrays of bicrystal junctions with high packing density was developed. Sub- $\mu \mathrm{m}$ junction arrays were fabricated and tested. We have shown that the width of the junctions is determined by optimal junction parameters and cannot be noticeably reduced or increased. Only the space between junctions could be made as small as possible.

An expression for calculation of the length of lumped arrays depending on critical current was suggested. For microwave investigations arrays embedded in CPS were fabricated and tested. The arrays with 431 junctions have shown frequency locking with an external irradiation. Voltage steps up to $20 \mathrm{mV}$ at liquid nitrogen temperature were demonstrated.

\section{REFERENCES}

[1] S. P. Benz and C. A. Hamilton, "A pulse-driven programmable Josephson voltage standard," Appl. Phys. Lett., vol. 68, pp. 3171-3173, May 1996.

[2] C. J. Burroughs, S. P. Benz, and P. D. Dresselhaus, "AC Josephson voltage standard error measurements and analysis," in CPEM'02 Conf. Dig., 2002, pp. 34-35.

[3] A. M. Klushin, W. Prusseit, E. Sodtke, S. I. Borovitskii, L. Amatuni, and H. Kohlstedt, "Shunted bicrystal Josephson junctions arrays for voltage standard," Appl. Phys. Lett., vol. 69, pp. 1634-1637, Sept. 1996.

[4] A. M. Klushin, R. Behr, K. Numssen, M. Siegel, and J. Niemeyer, "Accurate measurements of quantum voltage steps on arrays of bicrystal Josephson junctions," Appl. Phys. Lett., vol. 80, pp. 1972-1974, March 2002.

[5] A. M. Klushin, C. Weber, M. Darula, H. Kohlstedt, R. Semerad, and W. Prusseit, "Large critical current and current steps in shunted bicrystal Josephson junctions at nitrogen temperatures," Supercond. Sci. Technol., vol. 11, no. 11, pp. 609-613, July 1998.

[6] A. M. Klushin, S. I. Borovitskii, C. Weber, E. Sodtke, R. Semerad, W. Prusseit, V. D. Gelikonova, and H. Kohlstedt, "Programmable voltage standards based on HTS Josephson junction arrays," in Proc. EUCAS'97, vol. 1, 1997, pp. 587-590.

[7] C. Weber, A. M. Klushin, S. Beuven, A. van der Hart, H. Kohlstedt, R. Semerad, and W. Prusseit, "High temperature Josephson bicrystal junctions and arrays for metrological applications," IEEE Trans. Appl. Supercond., vol. 9, no. 2, pp. 4162-4165, June 1999.

[8] J. Hollkott, F. Kahlmann, C. Jaekel, S. Hu, B. Spangenberg, and H. Kurz, "Sub-100 nm lithography and high aspect-ratio masks for fabrication of Josephson devices by ion implantation," Microelectronic Engineering, vol. 41/42, pp. 403-406, 1998.

[9] Theva.. [Online]. Available: http://www.theva.com.

[10] A. M. Klushin, V. D. Gelikonova, K. Numssen, S. I. Borovitskii, and M. Siegel, "Microwave performance of bicrystal Josephson junction arrays,", to be published.

[11] H. Hilgenkamp and J. Mannhart, "Grain boundaries in high- $T \mathrm{c}$ superconductors," Rev. Mod. Phys., vol. 74, no. 2, pp. 485-550, April 2002.

[12] S. P. Benz, C. A. Hamilton, C. J. Burroughhs, and T. E. Harvey, "AC and dc bipolar standard using quantized pulses," IEEE Trans. Instr. Meas., vol. 48, pp. 266-269, April 1999.

[13] A. M. Klushin, A. Golubov, W. Prusseit, and H. Kohlstedt, "Comparative study of shunted bicrystal Josephson junctions," J. Low Temp. Phys., vol. 106, pp. 265-269, 1997.

[14] A. M. Klushin, Ph.D. dissertation, 1985.

[15] M. Schubert, T. May, G. Wende, L. Fritzsch, and H.-G. Meyer, "Coplanar strips for Josephson voltage standard circuits," Appl. Phys. Lett., vol. 79, no. 7, pp. 1009-1011, Aug. 2001. 